\title{
Rabbit Uterine Carcinoma
}

National Cancer Institute

\section{Source}

National Cancer Institute. Rabbit Uterine Carcinoma. NCI Thesaurus. Code C133883.

Uterine carcinoma that occurs in a rabbit. 